\title{
Pharmacognostic Studies of Selected Species of Curcuma L. a Medicinally Important Member of the Family Zingiberaceae
}

\author{
Jaladhar Pal ${ }^{1 *}$, Kumaresh Pal ${ }^{2}$ and Nirmalya Banerjee ${ }^{1}$ \\ ${ }^{1}$ Department of Botany, Visva-Bharati, Santiniketan 731235, West Bengal, India \\ ${ }^{2}$ Department of Biochemistry, Faculty of Agriculture, \\ Uttar Banga Krishi Viswavidyalaya, Pundi Bari 736165, West Bengal, India \\ *Corresponding author
}

\section{A B S T R A C T}

\begin{tabular}{|c|}
\hline Keywords \\
\hline $\begin{array}{l}\text { Foliar } \\
\text { micromorphology, } \\
\text { Organoleptic study, } \\
\text { Curcuma longa and } \\
\text { Curcuma zedoaria }\end{array}$ \\
\hline Article Info \\
\hline $\begin{array}{l}\text { Accepted: } \\
15 \text { April } 2020 \\
\text { Available Online: } \\
10 \text { May } 2020\end{array}$ \\
\hline
\end{tabular}

Present investigation deals with the pharmacognostic studies of some medicinally important species of Curcuma L. namely Curcuma amada Roxb., Curcuma aromatica Salisb., Curcuma caesia Roxb., Curcuma longa L. and Curcuma zedoaria (Christm.) Roscoe. Distinct variation was observed during foliar epidermal micromorphology study. Epidermal cell shape was found distinctly variable though cell wall outline was found to be straight in all the selected species. Leaves of the investigated taxa were found to be amphistomatic and the stomata are of tetracytic type. Stomatal index ranges from 13.54 (Curcuma longa) to 3.25 (Curcuma zedoaria). Trichomes were found to be present on both the epidermal surfaces of all the investigated taxa except Curcuma caesia where trichomes were found to be restricted to the upper epidermal surface. Petiole outline is almost concavo- convex in T.S. and the number of vascular bundle were ranges from 57 (Curcuma longa) to 39 (Curcuma caesia). Colour of the powdered crude drug varies from colourless to bright yellow. Textures of the fresh powders were smooth and fibrous. Moisture content ranged from $8.02 \%$ (Curcuma longa) to 14.53 (Curcuma amada). Total ash value ranged from $3.91 \%$ to $5.49 \%$. Microchemical colour reaction test of the ethanolic plant extracts showed the presence of important phytochemical groups like- alkaloids, flavonoids, proteins, saponins reducing sugars etc.

\section{Introduction}

Medicinal plants are the fundamental components of any indigenous medicine system. The use of different plant species for the treatment of different kinds of health hazards is the oldest form of health care system acknowledged in human civilization all over the globe (Fransworth, 1994), and presently more than $80 \%$ of the world populations, mostly of the third world countries depend on herbal medication for their primary health care (WHO, 1993). The genus Curcuma L. belonging to the family Zingiberaceae, originated in the Indo Malayan region (Purseglove, 1974) has a wide spread occurrence in the tropics of Asia to Africa and Australia. 
The genus globally consists of about 80 species of which 40 are reported from India (Sashikumar, 2005). The word Curcuma derived from the Arabic word "Kurkum" which means yellow colour, it is mainly due to the yellow colour of the underground rhizome. Curcuma is known as the golden spies of India.

The rhizome is vegetative in nature as well as the propagating part which is traditionally used in medicine and food since ancient times (Srimal, 1997). The use of different Curcuma species has been given in the vedic culture of India, nearly 4000 years back.

It reached China before the $7^{\text {th }}$ century, East Africa in the $8^{\text {th }}$ century and West Africa in the $13^{\text {th }}$ century. This multipurpose ancient and sacred spice of India, also known as 'Indian Saffron', finds a place in offerings on religious and ceremonial occasions (Khan et al., 2014).

India is the largest producer and exporter of turmeric in the world accounting for more than $50 \%$ of the world's trade; fulfilling $90 \%$ of world's demand (APEDA, 2018; Olojede et al., 2009).

Turmeric occupies about $6 \%$ of the total area under spices and condiments in India, and has considerable importance in Indian economy (Choudhury, 2018). Turmeric has already gained importance all over the world for therapeutic uses owing to its antiinflammatory, anti-diabetic, anticarcinogenic, anti-hepatotoxic, anti-viral, choleretic, antibiotic, anti-venomous and anti-rheumatic properties (Singh and Aggarwal, 2003; Wilken et al., 2011; Lin et al., 2008; Kaypee et al., 2015) and is frequently used in ayurvedic medicine of India as 'haridra'. Pharmacognostic, phytochemical and antimicrobial activity studies of medicinal plants have been carried out by many workers earlier for proper identification and standardization of natural products obtained from respective medicinal plants (Choudhury et al., 2012; Choudhury et al., 2013; Pal and Rahaman 2014; Ghosh and Rahaman 2015).

Though a good number of quality research has been undertaken on the therapeutic values and bioprospecting of Curcuma species mostly concentrated on the species Curcuma longa L. Research work relating to pharmacognostic, phytochemical studies of different species of the genus Curcuma L. are limited in number.

Therefore, the present investigation has been undertaken to study the pharmacognostic potential of these medicinal plant which will be very helpful in proper identification and standardization of the natural products obtained the plant.

\section{Materials and Methods}

Five medicinally important species of the family Zingeberaceae namely Curcuma amada Roxb., Curcuma aromatica Salisb., Curcuma caesia Roxb., Curcuma longa L. and Curcuma zedoaria (Christm.) Roscoe. have been selected for this study.

Plant specimens of those five selected taxa have been collected from the Department of Botany, University of North Bengal, West Bengal and have been identified by Prof. A. P. Das of the Department of Botany, North Bengal University.

Those plant specimens have been grown and maintained in the medicinal plant garden of Department of Botany, Visva-Bharati, Santiniketan, and the voucher specimens have been kept in Visva-Bharati Herbarium, Department of Botany, Visva- Bharati, Santiniketan, India for future reference. 
Scientific name, Local name and Medicinal uses of the selected plant species have been presented in Table 1.

\section{Epidermal micromorphology}

Leaf samples were cleared following the Bokhari's method (1970). The cleared leaf samples then mounted on the slide with a drop of $10 \%$ glycerine \& $1 \%$ aqueous safranin and observed under the compound light microscope.

\section{Vegetative anatomy}

Vegetative anatomy method was carried out following the standard methods of Johansen (1940).

\section{Preliminary microchemical screening}

Detection of different phytochemical groups was carried out by following different standard methods (Evans, 1996; Harbrone, 2002).

\section{Physical evaluation}

\section{Physical constant}

The physical evaluation of the powder was done following different methods (Peach and Tracy, 1955, Evans 2008) which includes determination of ash value and moisture content.

\section{Histochemical study}

Few drops of different reagents (Mayre's reagent, Wagnor's reagent, Lugols reagent, Phloroglucinol, lead acetate) were added to the thin sections of the rhizome of the investigated species and then those sections were observed under light microscope. These tests were done to detect different phytochemicals localized in different tissue zones of the rhizome (Trease and Evans, 1983).

\section{Results and Discussion}

General description and measurement of epidermal cells, stomata, microchemical colour reaction tests, physical parameters, histochemical localization tests of the investigated plant species were given below (Tables 2, 3, 4, 5 and Fig. 1-13).

\section{Foliar micromorphology}

Epidermal cell shape was found to be irregular in Curcuma amada, hexagonal in case of Curcuma aromatica and Curcuma longa though the shape was found to be polygonal in case of Curcuma caesia and Curcuma zedoaria. Cell wall outline was found to be straight in both the epidermal surfaces in all the selected species. Epidermal cell length was ranging from $82.61 \mu \mathrm{m}$ (upper surface of Curcuma longa) to $41.46 \mu \mathrm{m}$ (upper surface of Curcuma amada). Epidermal cell width was highest on upper surface of Curcuma aromatica $(40.34 \mu \mathrm{m})$ and lowest in case of upper surface of Curcuma amada $(24.67 \mu \mathrm{m})$. Epidermal cell frequency was found to be highest in upper surface of Curcuma amada $\left(977.69 / \mathrm{mm}^{2}\right)$ and lowest in lower surface of Curcuma zedoaria $\left(352.85 / \mathrm{mm}^{2}\right)$. Pallisade ratio was highest in Curcuma amada (14.21) and lowest in Curcuma caesia (6.2) (Table 2).

Stomatal features were also studied and all the investigated taxa showed amphistomatic type of stomata which are of tetracytic type. Stomatal length ranged from $38.13 \mu \mathrm{m}$ (upper surface of Curcuma zedoaria) to $19.32 \mu \mathrm{m}$ (lower surface of Curcuma amada) and the width of the stomatal ranges from $21.64 \mu \mathrm{m}$ (Curcuma aromatica upper surface) to $11.76 \mu \mathrm{m}$ (lower surface of Curcuma amada). Stomatal index was ranging from $13.54 \%$ 
(lower surface of Curcuma longa) to $3.25 \%$ (upper surface of Curcuma zedoaria). Highest stomatal frequency was found in the lower surface of Curcuma zedoaria $\left(163.52 / \mathrm{mm}^{2}\right)$ and lowest at both upper surface of Curcuma aromatica $\left(28.20 / \mathrm{mm}^{2}\right)$ (Table 3$)$.

Trichomes were found to be present on both the epidermal surfaces of all the investigated taxa except the lower surface of Curcuma caesia. Trichomes are of unicellular, non glandular type with swollen base and pointed tip in all the investigated taxa. Trichome length ranged from $218.2 \mu \mathrm{m}$ (upper surface of Curcuma aromatica) to $94.93 \mu \mathrm{m}$ (lower surface of Curcuma zedoaria).

Trichome frequency was highest on the lower surface of Curcuma longa $\left(13.45 / \mathrm{mm}^{2}\right)$ and lowest on the upper surface of Curcuma amada $\left(4.29 / \mathrm{mm}^{2}\right)$. Highest trichome index was found at upper surface of Curcuma amada (12.32\%) and lowest was at lower surface of Curcuma zedoaria (3.48\%) (Table 4).

\section{Petiole anatomy}

T.S. of the petiole revealed more or less similar type of histological features in petiole shape, outline and orientation of vascular bundle. Shape of the petiole is more or less concavo-convex in T.S. Petiole is surrounded by unicellular epidermal layer followed by a massive parenchymatous ground tissue, composed of thin walled cells.

Various numbers of air space were noted in the ground tissue. But, a distinct variation was recorded in the number of vascular bundle in the T.S of the petiole.

Highest number of vascular bundle was observed in Curcuma longa (57 Nos.) and lowest number of vascular bundles were observed in Curcuma caesia (39 Nos).

\section{Organoleptic study of powdered plant parts}

Crude drugs obtained from the powdered rhizome pieces were evaluated with different sensory organs are tested for the colour, odour, taste and texture which are listed below (Table 5).

\section{Physical parameters}

Moisture contents were also studied among the investigated taxa and they were found to be very distinct. Lowest value of moisture content was found in case of Curcuma longa $(8.02 \%)$ and the highest value was observed in case of Curcuma amada (14.53\%). Total ash-value ranged from $3.91 \%$ to $5.49 \%$ and the value was very diverse among the investigated species. Lowest ash value was found in case of Curcuma zedoaria (3.91\%) and the highest value was observed in Curcuma aromatica (5.49\%) (Table 6).

\section{Microchemical colour reaction test}

Through microchemical colour reaction tests of the ehanolic plant extracts, important phytochemical groups like alkaloids, flavonoids, proteins, saponins etc have been detected which indicated the medicinal properties of the selected plant species (Table 7).

\section{Histochemical study}

Histochemical study has been carried out to detect various phytochemical groups localized in different tissue zones of the rhizomes. Different phytochemical groups like alkaloids, proteins, flavonoids, lignin, saponins etc. were found in different tissue zones of the rhizome. It has been found that the connective tissues and cortical zones are main active sites for synthesis of high content of different phytochemical groups (Table 8). 
Table.1 Materials and their medicinal importance incorporated in the present investigation (Anonymous 2005)

\begin{tabular}{|c|c|c|c|}
\hline $\begin{array}{l}\text { Sl } \\
\text { No }\end{array}$ & Scientific name & $\begin{array}{l}\text { Local } \\
\text { name }\end{array}$ & Medicinal importance of different plant parts \\
\hline 1 & Curcuma amada Roxb. & Aam aada & $\begin{array}{l}\text { Rhizome: Used to treat pruigo, sprains, itch, skin } \\
\text { disease, bronchitis, asthama, hic cough; crushed in } \\
\text { water and juice taken in empty stomach as } \\
\text { carminative and to improve appetite. } \\
\text { Root: Used to treat diarrhoea, gleet, lumbago, } \\
\text { scabies and ulcer on penis. }\end{array}$ \\
\hline 2 & aromatica & $\begin{array}{l}\text { Ban halud, } \\
\text { Gyan } \\
\text { churamani }\end{array}$ & $\begin{array}{l}\text { Rhizome: Used to treat scabies, eruption of small } \\
\text { pox, and headache, paste applied to affected parts } \\
\text { as antidotes to snake bite. } \\
\text { Root: Paste with Kujri oil as massage to cool } \\
\text { down high fever. } \\
\text { Leave: Decoction with honey given to treat } \\
\text { dropsy. }\end{array}$ \\
\hline 3 & Curcuma caesia Roxb. & $\begin{array}{l}\text { Kalo } \\
\text { halud, } \\
\text { Kala haldi }\end{array}$ & $\begin{array}{l}\text { Rhizome: Purifies blood; used as tonic to brain } \\
\text { and heart; used to treat leucoderma, piles, } \\
\text { bronchitis, asthama, tumours, tuberculous glands } \\
\text { on the neck, enlargement of the spleen, to check } \\
\text { leucorrhal and gonorrhoeal discharges. } \\
\text { Root: Fine paste of rhizomes and roots applied on } \\
\text { the affected parts on the body as an antidote to } \\
\text { snake bite. }\end{array}$ \\
\hline 4 & Curcuma longa $\mathrm{L}$. & $\begin{array}{l}\text { Halud, } \\
\text { Haldi }\end{array}$ & $\begin{array}{l}\text { Rhizome: Used to treat disease of the blood, } \\
\text { leucoderma, scabies, urinary discharges, } \\
\text { inflammation , ozonea, biliousness, dyspepsia, } \\
\text { elephantiasis, small pox, swellings, boils, catarrh, } \\
\text { purulent opthalmia, diarrhoea, interminnet fever, } \\
\text { dropsy, bronchitis, paste with mustard oil applied } \\
\text { on skin disease. } \\
\text { Leave: Juice mixed with water given twice daily } \\
\text { to treat dysentery. }\end{array}$ \\
\hline 5 & $\begin{array}{l}\text { Curcuma zedoaria } \\
\text { (Christm.) Roscoe }\end{array}$ & Sathi, Palo & $\begin{array}{l}\text { Rhizome: Used to treat asthama, bronchitis, piles, } \\
\text { leucoderma, tumours, tuberculous glands on the } \\
\text { neck, enlarged spleen, gripping of children, pains } \\
\text { inflammation, tooth aches, bruises, sprains ; to } \\
\text { cure weakness after childbirth; to purify blood; } \\
\text { given to treat intestinal worms of children. } \\
\text { Root: Decoction taken to treat diarrhoea and piles. } \\
\text { Leave: Used to treat dropsy. }\end{array}$ \\
\hline
\end{tabular}


Table.2 Epidermal cell characters of the investigated plant species

\begin{tabular}{|c|c|c|c|c|c|c|c|}
\hline $\begin{array}{l}\text { Investigated } \\
\text { taxa }\end{array}$ & $\begin{array}{c}\text { Leaf } \\
\text { surface }\end{array}$ & Cell shape & $\begin{array}{c}\text { Cell } \\
\text { length } \\
(\mu \mathrm{m})\end{array}$ & $\begin{array}{c}\text { Cell } \\
\text { width } \\
(\mu \mathrm{m})\end{array}$ & $\begin{array}{c}\text { Cell } \\
\text { frequency } \\
\left(\text { No./mm }{ }^{2}\right)\end{array}$ & $\begin{array}{l}\text { Cell wall } \\
\text { out line }\end{array}$ & Palisade ratio \\
\hline \multirow[t]{2}{*}{ C. amada } & Upper & Irregular & 41.46 & 24.67 & 977.69 & Straight & \multirow[t]{2}{*}{14.21} \\
\hline & Lower & Irregular & 43.65 & 29.72 & 770.84 & Straight & \\
\hline \multirow[t]{2}{*}{ C. aromatica } & Upper & Hexagonal & 61.12 & 40.34 & 405.58 & Straight & \multirow[t]{2}{*}{12.5} \\
\hline & Lower & Hexagonal & 57.83 & 37.91 & 456.13 & Straight & \\
\hline \multirow[t]{2}{*}{ C. caesia } & Upper & Polygonal & 46.27 & 29.28 & 738.12 & Straight & \multirow[t]{2}{*}{6.2} \\
\hline & Lower & Polygonal & 49.18 & 33.27 & 611.16 & Straight & \\
\hline \multirow[t]{2}{*}{ C. longa } & Upper & Hexagonal & 52.41 & 30.1 & 633.89 & Straight & \multirow[t]{2}{*}{11.73} \\
\hline & Lower & Hexagonal & 48.73 & 34.37 & 597.06 & Straight & \\
\hline \multirow[t]{2}{*}{ C. zedoaria } & Upper & Polygonal & 82.61 & 31.07 & 389.60 & Straight & \multirow[t]{2}{*}{7.2} \\
\hline & Lower & Polygonal & 79.81 & 35.51 & 352.85 & Straight & \\
\hline
\end{tabular}

Table.3 Stomatal features of the investigated species

\begin{tabular}{|c|c|c|c|c|c|c|}
\hline $\begin{array}{l}\text { Investigated } \\
\text { taxa }\end{array}$ & $\begin{array}{l}\text { Leaf } \\
\text { surface }\end{array}$ & $\begin{array}{c}\text { Stomatal } \\
\text { type }\end{array}$ & $\begin{array}{c}\text { Stomatal } \\
\text { length }(\mu \mathrm{m})\end{array}$ & $\begin{array}{c}\text { Stomatal } \\
\text { width }(\mu \mathrm{m})\end{array}$ & $\begin{array}{c}\text { Stomatal index } \\
(\%)\end{array}$ & $\begin{array}{l}\text { Stomatal } \\
\text { frequency } \\
\left(\text { No. } / \mathrm{mm}^{2}\right)\end{array}$ \\
\hline \multirow[b]{2}{*}{ C. amada } & Upper & Tetracytic & 27.44 & 12.34 & 8.10 & 61.01 \\
\hline & Lower & Tetracytic & 19.32 & 11.76 & 13.40 & 76.04 \\
\hline \multirow{2}{*}{ C. aromatica } & Upper & Tetracytic & 36.62 & 21.64 & 6.08 & 28.20 \\
\hline & Lower & Tetracytic & 31.33 & 20.07 & 7.44 & 35.67 \\
\hline \multirow[t]{2}{*}{ C. caesia } & Upper & Tetracytic & 30.06 & 15.64 & 9.55 & 60.60 \\
\hline & Lower & Tetracytic & 28.63 & 13.72 & 12.26 & 66.75 \\
\hline \multirow[t]{2}{*}{ C. longa } & Upper & Tetracytic & 36.20 & 20.31 & 11.11 & 51.36 \\
\hline & Lower & Tetracytic & 34.69 & 19.77 & 13.54 & 63.23 \\
\hline \multirow[t]{2}{*}{ C. zedoaria } & Upper & Tetracytic & 38.13 & 14.88 & 3.25 & 146.39 \\
\hline & Lower & Tetracytic & 34.76 & 19.72 & 7.16 & 163.52 \\
\hline
\end{tabular}

Table.4 Trichome features of the investigated taxa

\begin{tabular}{|c|c|c|c|c|c|}
\hline $\begin{array}{l}\text { Investigated } \\
\text { taxa }\end{array}$ & $\begin{array}{l}\text { Leaf } \\
\text { surface }\end{array}$ & Trichome type & $\begin{array}{l}\text { Trichome } \\
\text { size }(\mu \mathrm{m})\end{array}$ & $\begin{array}{l}\text { Trichome } \\
\text { frequency } \\
\left(\text { No./ } / \mathrm{mm}^{2}\right)\end{array}$ & $\begin{array}{l}\text { Trichome } \\
\text { Index }(\%)\end{array}$ \\
\hline \multirow[t]{2}{*}{ C. amada } & Upper & \multirow{2}{*}{$\begin{array}{l}\text { Nonglandular, unicellular with } \\
\text { swollen base and pointed tip }\end{array}$} & 157 & 4.29 & 6.38 \\
\hline & Lower & & 164.8 & 12.31 & 12.32 \\
\hline \multirow[t]{2}{*}{ C. aromatica } & Upper & \multirow{2}{*}{$\begin{array}{l}\text { Nonglandular, unicellular with } \\
\text { swollen base and pointed tip }\end{array}$} & 218.2 & 6.42 & 4.86 \\
\hline & Lower & & 157.3 & 10.14 & 8.54 \\
\hline \multirow[t]{2}{*}{ C. caesia } & Upper & $\begin{array}{l}\text { Nonglandular, unicellular with } \\
\text { swollen base and pointed tip }\end{array}$ & 131.73 & 11.79 & 5.2 \\
\hline & Lower & Absent & ------- & -------- & -------- \\
\hline \multirow[t]{2}{*}{ C. longa } & Upper & \multirow{2}{*}{$\begin{array}{l}\text { Nonglandular, unicellular with } \\
\text { swollen base and pointed tip }\end{array}$} & 164.37 & 9.34 & 7.64 \\
\hline & Lower & & 132.82 & 13.45 & 6.51 \\
\hline \multirow[t]{2}{*}{ C. zedoaria } & Upper & \multirow{2}{*}{$\begin{array}{l}\text { Nonglandular, unicellular with } \\
\text { swollen base and pointed tip }\end{array}$} & 104.89 & 5.89 & 4.97 \\
\hline & Lower & & 94.93 & 4.72 & 3.48 \\
\hline
\end{tabular}


Table.5 Findings of the organoleptic study among the investigated taxa

\begin{tabular}{|l|l|l|l|l|l|}
\hline $\begin{array}{l}\text { Investigated } \\
\text { taxa }\end{array}$ & $\begin{array}{c}\text { Plant } \\
\text { parts }\end{array}$ & \multicolumn{1}{|c|}{ Colour } & \multicolumn{1}{|c|}{ Odour } & Taste & Texture \\
\hline C. amada & Rhizome & Pale yellow & $\begin{array}{l}\text { Fresh, slightly rosy with a fruity } \\
\text { top note of raw mangoes }\end{array}$ & Sweet, mildly spicy & Smooth \\
\hline C. aromatica & Rhizome & $\begin{array}{l}\text { Greenish } \\
\text { brown }\end{array}$ & $\begin{array}{l}\text { Pungent, warm, woody-rooty } \\
\text { with a camphory top note }\end{array}$ & $\begin{array}{l}\text { Bitter, flat earthy } \\
\text { and mildly spicy }\end{array}$ & Fibrous \\
\hline C. caesia & Rhizome & $\begin{array}{l}\text { Colour } \\
\text { less, } \\
\text { become } \\
\text { blue on } \\
\text { exposure to } \\
\text { air }\end{array}$ & $\begin{array}{l}\text { Warm, mildly spicy, } \\
\text { camphoraceous with woody top } \\
\text { note }\end{array}$ & $\begin{array}{l}\text { Slightly bitter, Fibrous } \\
\text { warm with a spicy } \\
\text { after taste }\end{array}$ & Fibath \\
\hline C. longa & Rhizome & $\begin{array}{l}\text { Bright } \\
\text { yellow }\end{array}$ & $\begin{array}{l}\text { Spicy, mildly earthy, smooth } \\
\text { warm with a rooty top note }\end{array}$ & $\begin{array}{l}\text { Pleasantly warm } \\
\text { with a spicy after } \\
\text { taste }\end{array}$ & Smooth \\
\hline C. zedoaria & Rhizome & $\begin{array}{l}\text { Light } \\
\text { yellow }\end{array}$ & $\begin{array}{l}\text { Warm, penetrating, pleasant, } \\
\text { camphoraceous with a flowery } \\
\text { top note }\end{array}$ & $\begin{array}{l}\text { Slightly bitter with } \\
\text { a spicy after taste }\end{array}$ & Smooth \\
\hline
\end{tabular}

Table.6 Comparison of ash value and moisture content of the investigated taxa

\begin{tabular}{|c|c|c|c|c|c|}
\hline \multirow[t]{2}{*}{ Investigated taxa } & \multicolumn{4}{|c|}{ Ash value } & \multirow{2}{*}{$\begin{array}{l}\text { Moisture content } \\
\text { Loss on drying (\%) }\end{array}$} \\
\hline & Total ash $(\%)$ & $\begin{array}{l}\text { Water } \\
(\%)\end{array}$ & soluble ash & $\begin{array}{l}\text { Acid insoluble ash } \\
(\%)\end{array}$ & \\
\hline C. amada & 5.19 & & 1.28 & 1.06 & 14.53 \\
\hline C. aromatica & 5.49 & & 1.37 & 2.07 & 9.37 \\
\hline C. caesia & 3.99 & & 0.96 & 1.83 & 12.43 \\
\hline C. longa & 4.08 & & 1.58 & 2.02 & 8.02 \\
\hline C. zedoaria & 3.91 & & 1.42 & 1.07 & 9.19 \\
\hline
\end{tabular}

Table.7 Microchemical tests of the investigated species

\begin{tabular}{|l|l|l|c|c|c|c|c|}
\hline Test/ Reagent & Test for & Nature of change & $\begin{array}{c}\text { C. } \\
\text { amada }\end{array}$ & $\begin{array}{c}\text { C. } \\
\text { aromatica }\end{array}$ & $\begin{array}{c}\text { C. } \\
\text { caesia }\end{array}$ & C. longa & $\begin{array}{c}\text { C. } \\
\text { zedoaria }\end{array}$ \\
\hline $\begin{array}{l}\text { Dragendroff's } \\
\text { reagent }\end{array}$ & Alkaloids & Orange brown ppt & + & ++ & ++ & ++ & ++ \\
\hline Wagner's reagent & Alkaloids & Orange brown ppt & ++ & + & + & + & ++ \\
\hline Mayer's reagent & Alkaloids & White/cream ppt & + & ++ & ++ & ++ & ++ \\
\hline Shinoda test & Flavonoids & Magenta colour & + & -- & + & + & -- \\
\hline $\mathbf{1 0 \% ~ N a O H}$ & Flavonoids & Yellow colour & + & + & + & ++ & - \\
\hline Salkowski test & $\begin{array}{l}\text { Steroids and } \\
\text { Triterpenoids }\end{array}$ & $\begin{array}{l}\text { Reddish blue and } \\
\text { green fluorescence }\end{array}$ & ++ & ++ & -- & ++ & + \\
\hline $\begin{array}{l}\text { Benedict's } \\
\text { reagent }\end{array}$ & Reducing sugars & Brick red ppt & -- & + & + & ++ & ++ \\
\hline
\end{tabular}




\begin{tabular}{|l|l|l|c|c|c|c|c|}
\hline Fehling's reagent & Reducing sugars & Brick red ppt & -- & + & + & ++ & + \\
\hline $\begin{array}{l}\text { Molish's test } \\
\mathbf{1 0 \%} \mathbf{K}_{\mathbf{2}} \mathbf{C r}_{2} \quad \mathbf{O}_{7}\end{array}$ & Tannins & Red violet ring & - & + & - & +++ & + \\
\hline $\begin{array}{l}\mathbf{5 \%} \text { FeClution } \\
\text { Solution }\end{array}$ & Tannins & Gellow brown ppt & + & ++ & ++ & ++ & ++ \\
\hline $\begin{array}{l}\mathbf{1 \%} \quad \text { lead acetate } \\
\text { solution }\end{array}$ & Saponins & White ppt & + & +++ & -- & + & + \\
\hline Bentrager's test & Anthraquinones & Pink colour & - & -- & -- & - & ++ \\
\hline Ninhydrine test & Amino acids & Purple colour & - & +++ & + & - & -- \\
\hline $\begin{array}{l}\text { Phloroglucinol } \\
\text { test }\end{array}$ & Lignin & Red colour & + & ++ & + & + & ++ \\
\hline Millon's reagent & Proteins & White ppt. & ++ & ++ & ++ & ++ & ++ \\
\hline Lugols & Proteins & Faint yellow colour & ++ & ++ & ++ & ++ & ++ \\
\hline
\end{tabular}

Table.8 Findings of the histochemical localization of the investigated taxa

\begin{tabular}{|c|c|c|c|c|c|c|}
\hline $\begin{array}{l}\text { Investigated } \\
\text { taxa }\end{array}$ & Alkaloid & Tannin & Protein & Lignin & Flavonoid & $\begin{array}{l}\text { Reducing } \\
\text { sugar }\end{array}$ \\
\hline C. amada & $\begin{array}{l}\text { Outer } \\
\text { cortex, few } \\
\text { cells of } \\
\text { inner } \\
\text { cortex, } \\
\text { vascular } \\
\text { bundle }\end{array}$ & $\begin{array}{l}\text { Outer } \\
\text { cortex, } \\
\text { xylem }\end{array}$ & $\begin{array}{l}\text { Few } \\
\text { cortical } \\
\text { cells and } \\
\text { phloem } \\
\text { tissue }\end{array}$ & Endodermis & $\begin{array}{l}\text { Pith and } \\
\text { phloem }\end{array}$ & $\begin{array}{l}\text { Xylem } \\
\text { tissue and } \\
\text { inner } \\
\text { cortical } \\
\text { cells }\end{array}$ \\
\hline C. aromatica & $\begin{array}{l}\text { Vascular } \\
\text { bundle }\end{array}$ & $\begin{array}{l}\text { Few cells } \\
\text { of cortex }\end{array}$ & $\begin{array}{l}\text { Epidermis, } \\
\text { few cells of } \\
\text { hypodermis } \\
\text { and cortex }\end{array}$ & $\begin{array}{l}\text { Not } \\
\text { detected }\end{array}$ & $\begin{array}{l}\text { Phloem } \\
\text { tissue }\end{array}$ & $\begin{array}{l}\text { Not } \\
\text { detected }\end{array}$ \\
\hline C. caesia & $\begin{array}{l}\text { Few cells of } \\
\text { xylem and } \\
\text { pith }\end{array}$ & $\begin{array}{l}\text { Pith and } \\
\text { cortex } \\
\text { cells }\end{array}$ & Xylem cells & $\begin{array}{l}\text { Vascular } \\
\text { bundles }\end{array}$ & $\begin{array}{l}\text { Few cells } \\
\text { of xylem }\end{array}$ & Pith zone \\
\hline C. longa & $\begin{array}{l}\text { Few cells of } \\
\text { pith and } \\
\text { cortex }\end{array}$ & $\begin{array}{l}\text { Few cells } \\
\text { of pith } \\
\text { and } \\
\text { cortex }\end{array}$ & $\begin{array}{l}\text { Few cells of } \\
\text { pith and } \\
\text { cortex }\end{array}$ & $\begin{array}{l}\text { Few cells } \\
\text { of pith and } \\
\text { cortex }\end{array}$ & $\begin{array}{l}\text { Few cells } \\
\text { of pith and } \\
\text { cortex }\end{array}$ & $\begin{array}{l}\text { Few cells } \\
\text { of xylem } \\
\text { tissue }\end{array}$ \\
\hline C. zedoaria & $\begin{array}{l}\text { Outer } \\
\text { cortex and } \\
\text { xylem } \\
\text { tissue }\end{array}$ & $\begin{array}{l}\text { Few cells } \\
\text { of xylem, } \\
\text { pith and } \\
\text { cortex }\end{array}$ & $\begin{array}{l}\text { Vascular } \\
\text { bundle }\end{array}$ & $\begin{array}{l}\text { Endodermis } \\
\text { and xylem } \\
\text { tissue }\end{array}$ & $\begin{array}{l}\text { Phloem } \\
\text { zone }\end{array}$ & $\begin{array}{l}\text { Few cells } \\
\text { of cortex } \\
\text { and } \\
\text { endodermis }\end{array}$ \\
\hline
\end{tabular}




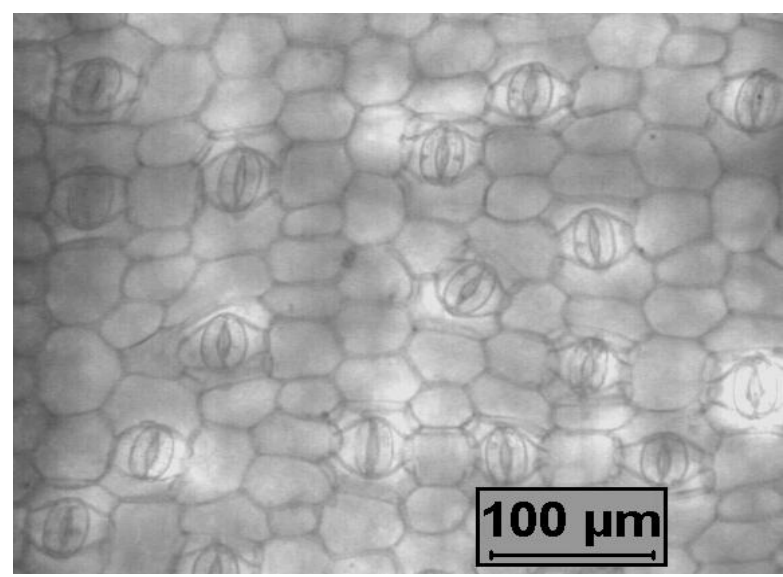

Fig.1 Foliar epidermal micromorphology of Curcuma aromatica

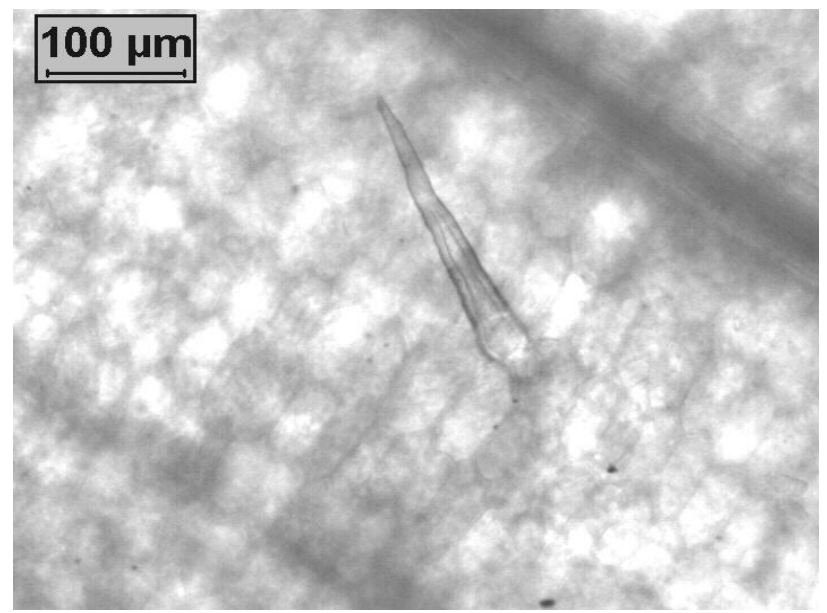

Fig.3 Trichomes of Curcuma zedoaria

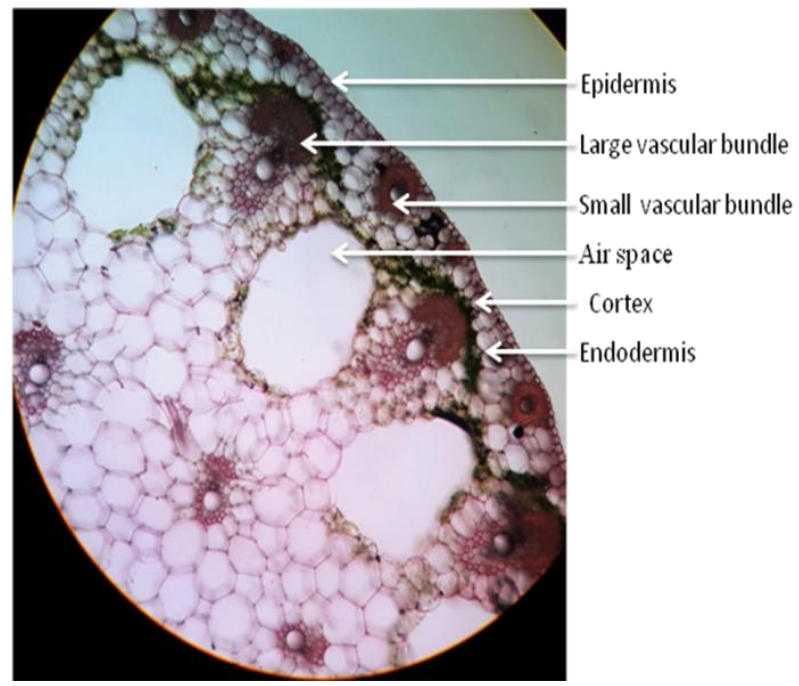

Fig.5 T.S. through the petiole of Curcuma amada

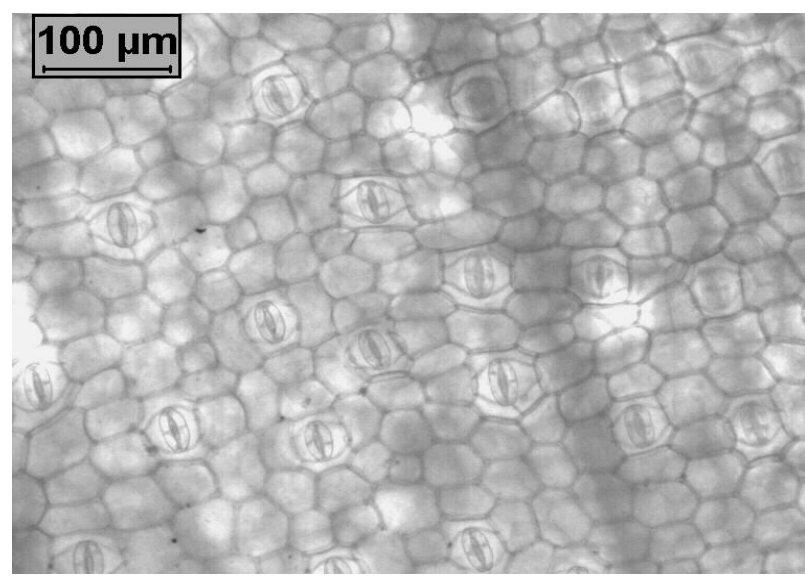

Fig.2 Foliar epidermal micromorphology of Curcuma caesia

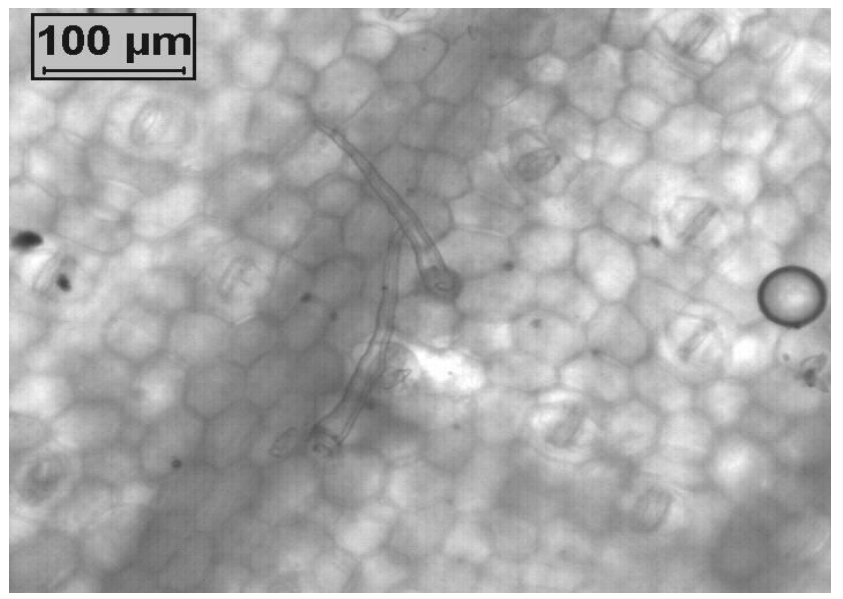

Fig.4 Trichomes of Curcuma aromatica

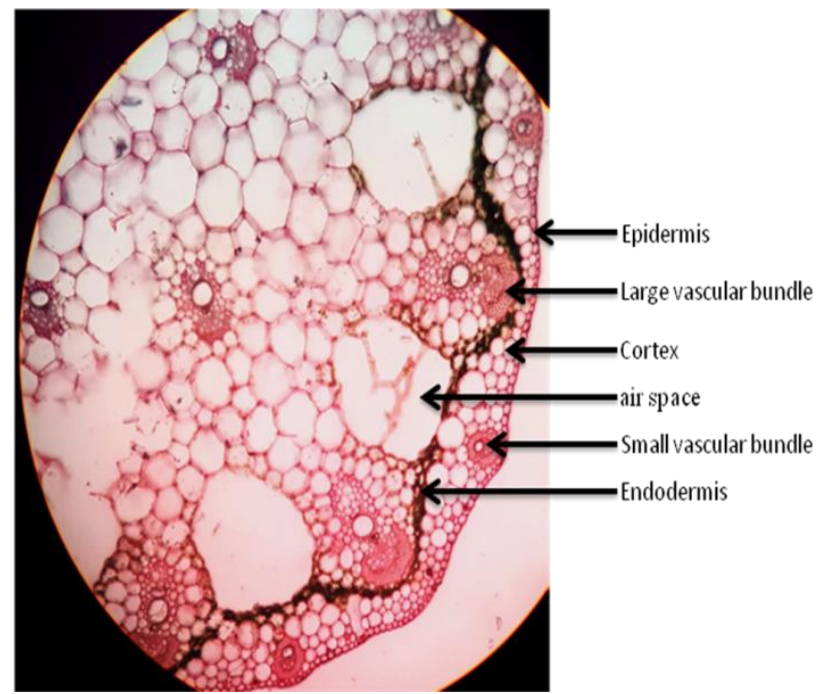

Fig.6 T.S. through the petiole of Curcuma aromatica 


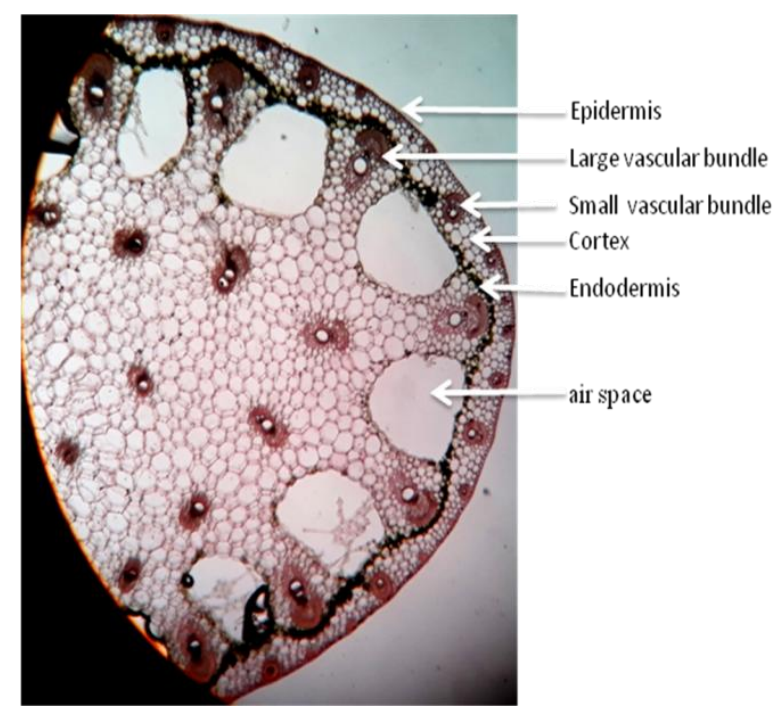

Fig.7 T.S. through the petiole of Curcuma

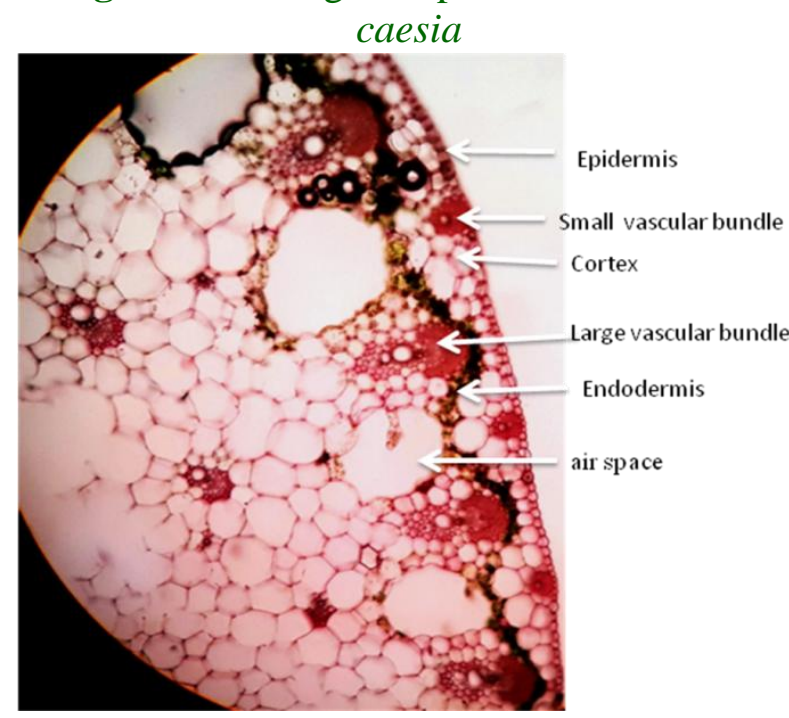

Fig.9 T.S. through the petiole of Curcuma zedoaria

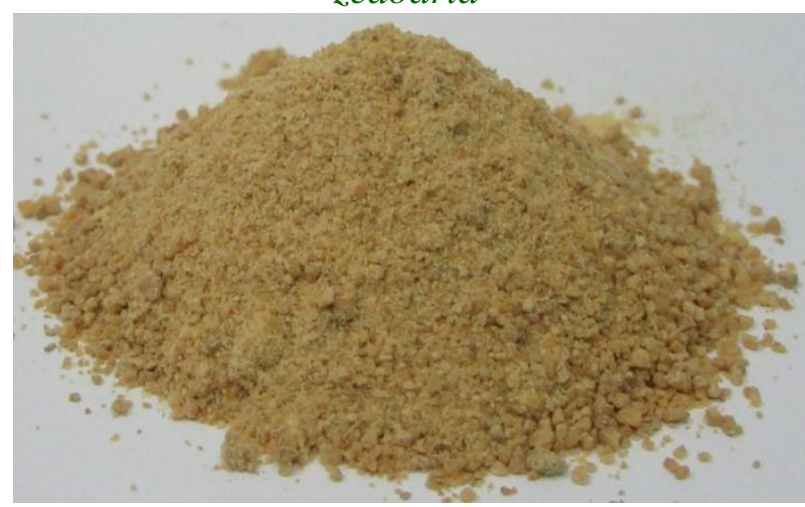

Fig.11 Crude drug from Curcuma aromatica Rhizome

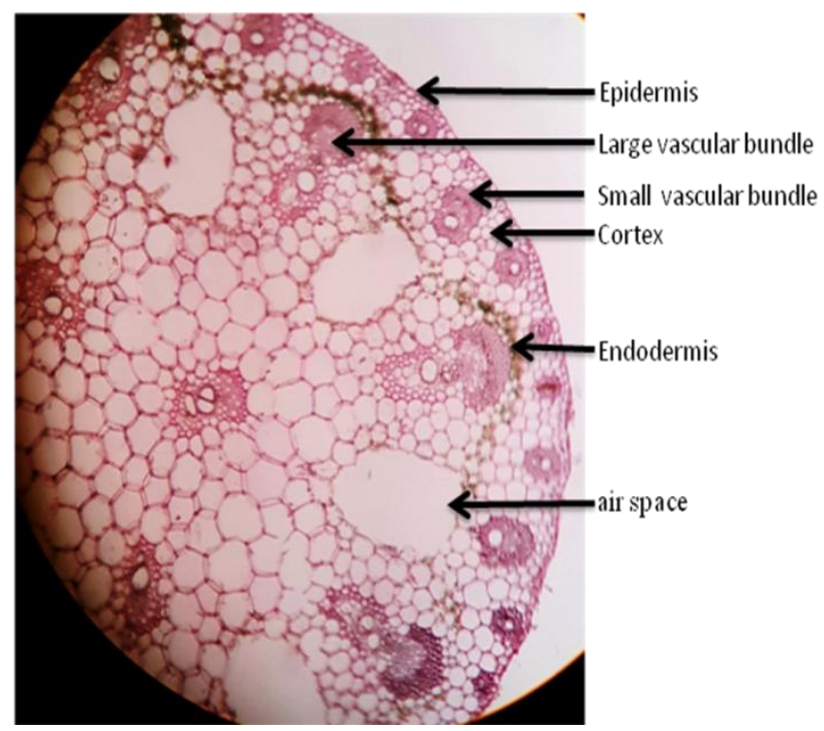

Fig.8 T.S. through the petiole of Curcuma longa

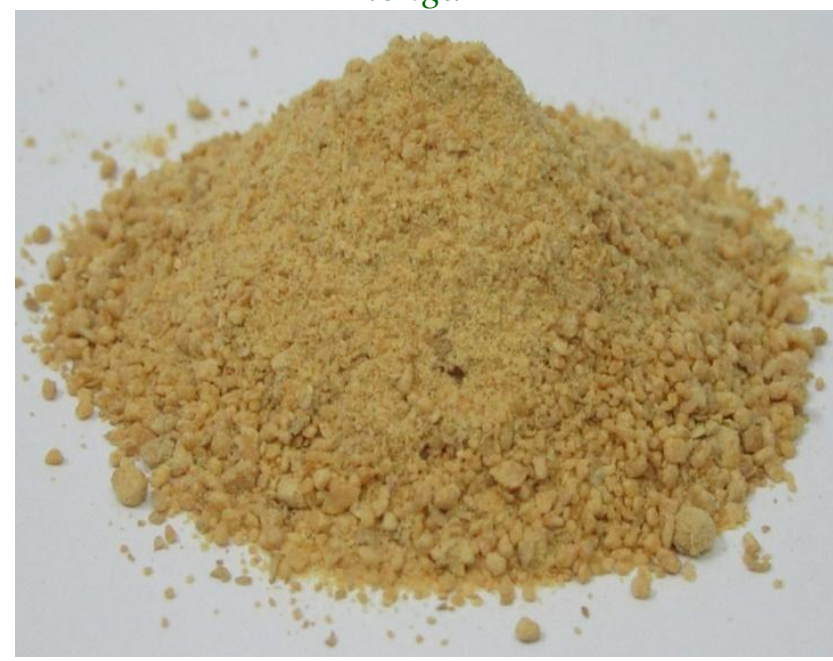

Fig.10 Crude drug from Curcuma amada Rhizome

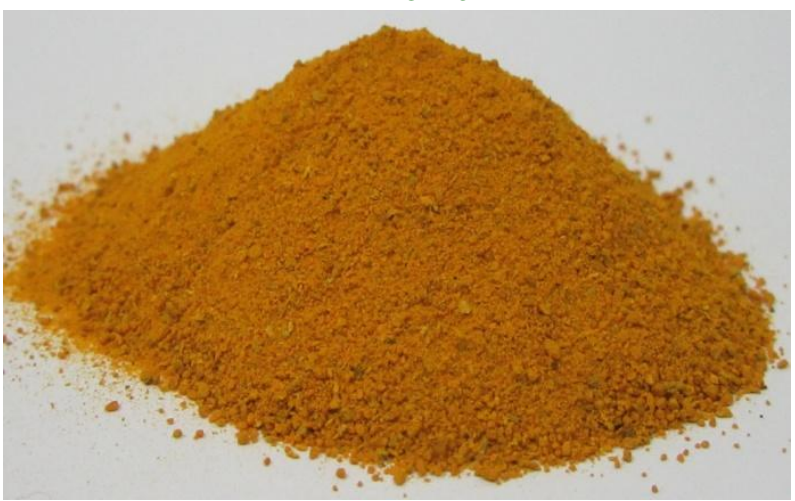

Fig.12 Crude drug from Curcuma longa Rhizome 


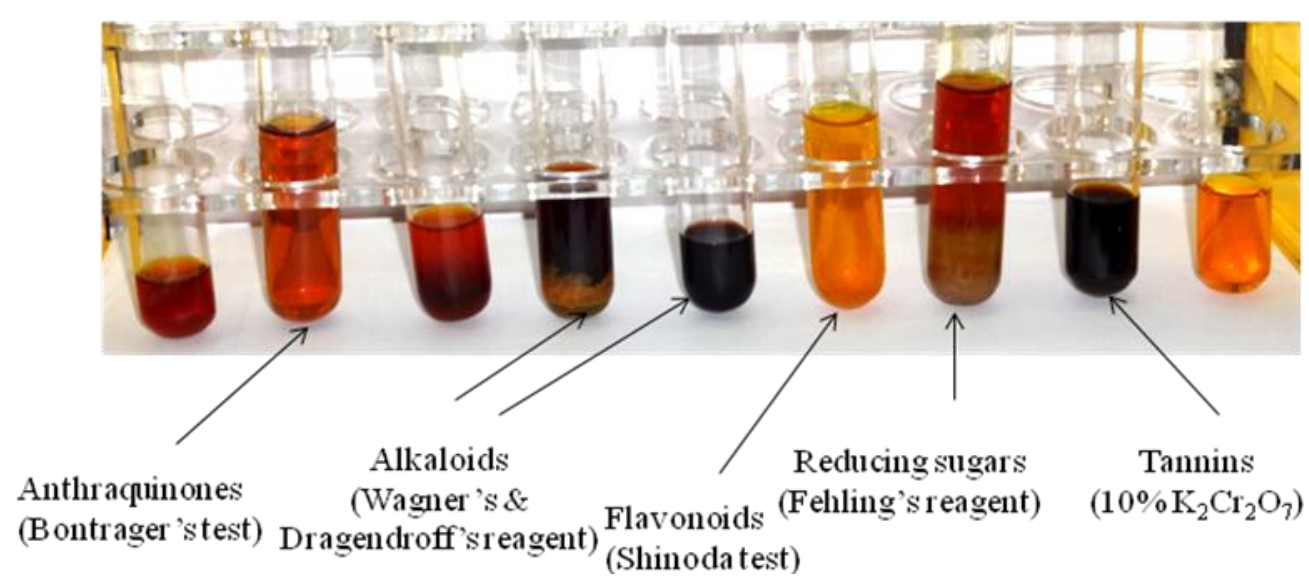

Fig.13 Microchemical colour reaction test of ethanolic extracts of Curcuma longa (Rhizome)

Total ash value is very diverse among the investigated species. Lowest ash value was recorded in Curcuma zedoaria $(3.91 \%)$ and the highest value was observed in Curcuma aromatica $(5.49 \%)$.

These results indicate that the selected plant species are rich in mineral contents. Acid insoluble and water soluble ash determination is important, because it indicates the quality and purity of the crude drug (Dev et al., 2015). Hence the physical parameters indicate the therapeutic potential of the crude drugs obtained from the selected plant species.

The phytochemical groups like alkaloids, flavonoids, proteins, saponins are present among the investigated species. A variety of biological activities like antioxidant, CNS stimulatory, antihelminthic, anti-hypertensive, anti-malarial, anti-diabetic, anti-rheumatic, anti-cancerous, anti-inflammatory, antimicrobial effects of various types of phytochemical groups have been critically studied by many workers earlier (Sinha et al., 2013; Obouayeba et al., 2014; Kumar et al., 2012; Hegde et al., 2010; Pal et al., 2020; Prakash et al., 2009). Flavonoids from different plant sources have been reported to have anti-inflammatory, anti-arthritic and anti-oxidant activities. Plants containing high amount of flavonoids were found to have more therapeutic values (Nagarkar et al., 2013). Through phytochemical screening it has been found that the selected plant species are of possessing adequate quantity of flavonoids.

From the above mentioned findings following key to the identification of the investigated taxa may be deduced

1. Trichomes are restricted to the upper epidermal surfaces ........ 2

2. Epidermal cells are irregular in shape $C$. amada

2. Epidermal cells are polygonal in shape $C$. caesia

1. Trichomes are present on both the epidermal surfaces ....... 3

3. Epidermal cells are hexagonal in shape. 4

4. In petiole T.S., number of vascular bundle 50 and number of air space $15 C$. aromatica

4 In petiole T.S., number of vascular bundle 57 and number of air space $16 \ldots \ldots . C$. longa

3. Epidermal cells are polygonal in shape C. zedoaria 
This study will be very useful in proper identification of the selected plant species with the key described. It will be also useful for proper identification of crude drugs obtained from these selected genera of Curcuma L. along with detection of drug adulterants. Thus it will be a tool in maintaining the quality of crude drugs obtained from the selected plant species. Moreover, the pharmacognostic studies of the investigated taxa highlight the presence of some important phytochemical groups which indicate promising curative potentials of these medicinal plants which need further investigation to explore the novel bioactive compounds. Thus it can be concluded that the crude drug of the selected plant species are good candidates for bioprospecting.

\section{Acknowledgements}

Authors are grateful to the Department of Botany, Visva-Bharati (A Central University) for providing necessary laboratory facilities and The University Grants Commission, New Delhi for providing the financial assistance to the first author in the form of UGC BSR Fellowship (Ref No. F.7 220/2009 dated 13.12.2012)

\section{References}

Adeyeye, E.I. and Ayejuyo, O.O. 1994. Chemical composition of Cola accuminata and Grarcinakola seed grown in Nigeria. Int. J. Food Sci Nutri. 45, 223-230.

Ahmed, K.J. 1979. Stomatal features of Acanthaceae. In: Sen DN (ed) Structure, Function and Ecology of Stomata. Bishen Sing Mahendra Pal Sing; Dehradun, India. Pp. 43-60.

Anonymous. (2005). Medicinal Plant resources of South West Bengal, (Research Wing, Directorate of Forests, Govt. of West Bengal,
Directorate of Forests, Govt. of West Bengal., Vol-I, pp.198.)

APEDA, 2018.Agricultural and Processed Food Products Export Development Authority (APEDA), New Delhi, India. APEDA-AgriXchange Profile of Turmeric Retrieved on 1 June, 2018. http://agriexchange.apeda.gov.in.

Bierhorst, D.W. 1960. Observation on tracheary elements, Phytomorphology. 10, 249-305.

Bokhari, M. H., 1970. Morphology and taxonomic significance of foliar sclerids in Limonium. Notes Royal Bot. Gard. 30, 43-53.

Choudhary,A.K., Rahi,S.2018. Organic cultivation of high yielding turmeric (Curcuma longa L.) cultivars: a viable alternative to enhance rhizome productivity, profitability, quality and resource-use efficiency in monkeymenace areas of north-western Himalayas. Ind. Crops Prod. 124, 495-504.

Choudhury, S., Rahaman, C. H. and Mandal, S. 2013. Pharmacognostic studies of Ampelocissus latifolia (Roxb.) Planch - An important ethnomedicinal plant. International J. of Current Research. 5(3), 643-648.

Choudhury, S., Rahaman, C.H. and Mandal, S.2009b. Studies on leaf epidermal micromorphology, wood element characters and phytochemical screening of three medicinally important taxa of the family Convolvulaceae. J. Environ. and Sociobiol. 6 (2), 105-118.

Cutler, D. F. 1984. Systemic anatomy and embryology, recent development. In: Heywood VH, Moore DM (eds) Current concepts in Plant Taxonomy, Academic Press: London, UK, Pp. 107-131 .

Dev, U.K., Hossain, T. M. and Islam, Z.M. 
2015. Phytochemical investigation, antioxidant activity and antihelmintic activity of Mikania micrantha leaves. World J. Pharma. Res. 4(5), 121-133.

Evans, W. C. 1996. Trease and Evan's Pharmacognosy. $14^{\text {th }}$ edn. W.B. Saunders Comp. Ltd.

Fahn, A. 1990. Plant anatomy, Pergamon press: New York. $4^{\text {th }}$ edn.

Farnsworth, N.R. 1994. Ethnopharmacology and new drug development. In: Chadwick DJ, Marsh J (eds) Ethnobotany and the search for new drugs: Ciba Foundation Symposium, Chichester, UK: Wiley, Pp. 42-59.

Fatima, A., Singh PP, Agarwal, P., Irchhaiya, R., Alok, S., and Amita, V.2013.Treatment of various diseases by Carissa spinarum L. - a promising shrub; Intl. J. Pharma Sci \& Res. 4(7), 2489-2495.

Ghosh, P. and Rahaman, C.H. 2015. Pharmacognostic, Phytochemical and Antioxidant Studies of Adenanthera pavonina L. Intl. J. Pharmacog. \& Phytochem Res. 7(1), 30-37.

Gogoi, R., Bokolial, D., Das, D.S. 2002. Leaf epidermal morphology of some species of Zingiberaceae. Plant Archives. 2, 257-262.

Harborne, J. B. 2002.Phytochemical Methods. 5 th edn. Chapman \& Hall, London.

Hegde, V., Nagarkatti, M., and Nagarkatti, P.S. 2010. Cannabinoid receptor activation leads to massive mobilization of myeloid-derived suppressor cells with potent immunosuppressive properties; Europ. J. Immunology. 40 (12), 3358-71.

Hussain, S.P., Hofseth, L.J. and Harris, C.C. 2003. Radical causes of cancer, Nat, Revw Cancer. 3, 276 -285.

Ibegbu, A.O., Okonji, U.J., Hamman, W.O., Umana, U.E., Ikyembe, D.T. and Musa, S.A. 2012. Antiinflammatory effects of the aqueous extracts of plantain roots (Musa Species). Bri J Pharmacol \& Toxicol. 3(2), 70-75.

Inamder, J. A. 1970. Epidermal Structure and ontogeny of Caryophyllaceous stomata in some Acanthaceae. Bot. Gaz. 131, 261-268.

Johansen, D. A. 1940. Plant Microtechnique, McGraw-Hill, New York.

Kaypee, S., Mandal, S., Chatterjee, S., Kundu, T.K. 2015. Emerging Epigenetic Therapies: Lysine Acetyltransferase Inhibitor Epigenetic Cancer Therapy (Steven Gray Edited) 978-0-12-800206-3 Elsvier Publication.

Khan, A.R., Khan, M.R., Sahreen, S., and Ahmed, M. 2012. Evaluation of phenolic contents and antioxidant activity of various solvent extracts of Sonchus asper (L.) Hill; Chem. Cent. J. 6(12), 212-218.

Khan, M.H., Choudhary, A.K., Rahi, S. 2014.Production technology of turmeric (Curcuma longa L.). In: Choudhary (Ed.), Advances in Vegetable Agronomy. ICAR Publication, New Delhi, India, Pp. 198-201.

Kokoski, J., Kokoski, R. and Salma, F. J. 1958. Fluorescence of powder vegetable drugs under ultraviolate radiation. J. Am. Pharm. Asso. 47(10), 715-717.

Kothari M.J. and Shah G.L. 1975. Epidermal structure and ontogeny of stomata in the Papilionaceae. Bot. Gaz. 136, 372379.

Kumar, M., and Garg, R. 2012.Phytochemical Properties and Antioxidant Activity of Hibiscus sabdariffa Linn; Intl. J. Pharmacy \& Chem Sci. 1 (3), 12361240.

Leelavathi, P.M. and Ramayya, N. 1983. Structure, distribution and classification of plant

Lin, S.S, Huang, H., Yang, J., Chung, J. 2008. 
DNA damage and endoplasmic reticulum stress meditated curcumininduced cell cycle arrest and apoptosis in human lung carcinoma A-549 cells through the activation caspases cascade- and mitochondrial-dependent pathway. Cancer Lett. 272(1), 77-90.

Metcalfe, C.R. and Chalk, L. 1950. Anatomy of Dicotyledons, Vol. 1, Clarendon Press, Oxford,UK.

Nagarkar, B., Jagtapati, S., Nirmal, P., Narkhede, A., Kuvalekar, A., Kulkarni, O., and Harsulkar, A. 2013. Comparative evaluation of antiinflammatory potential of medicinally important plants; Intl. J. Pharmacy \& Pharmaceutical Sci. 5(3), 239-243.

Obouayeba, A.P., Latte, T., Okoma, M., Diabate, S. and Kouakou, T.H. 2014. Phytochemical and Antioxidant Activity of Roselle (Hibiscus sabdariffa L.) Petal Extracts; Res. J. Pharma, Biol. \& Chem. Sci. 5(2), 1453-1465.

Ogundipe, O.T. and Olatunji, O.A. 1991. The leaf anatomy of the species of Cochlospermum Kunth. (Cochlospermaceae) in West Africa. Feddes Repertorium. 102, 183-187.

Olojede, A. O., Nwokocha, C. C., Akinpelu, A. O. and Dalyop, T,2009.Effect of variety, rhizome and seed bed types on yield of turmeric (Curcuma longa L.) under a humid tropical agroecology.Advan.Biol. Res. 3(1-2), 4042.

Pal, K. and Rahaman, C.H. 2014. Studies on foliar epidermal micromorphology, vegetative anatomy and xylem elements of four members of Portulacaceae, Intl. J. Curr. Res. 6(02), 4968-4975.

Pal, K., Chowdhury, S., Dutta, S.K., Chakroborty, S., Chakroborty, M., Pandit, G.K., Dutta, S., Paul, P.K., Chowdhury, A., Majumder, B.,
Sahana, N., Mandal., S.2020. Analysis of Rhizome colour content, bioactive compound profiling and ex-situ conservation of turmeric genotypes (Curcuma longa L.) from subHimalayan terai region of India. Industrial crops and products 150,112401 .

Pant, D.D. and Mehera, B. 1963: Development of Caryophyllaceous stomata in Asteracantha longifolia Nees, Ann. Bot. 27, 647-652.

Peach and Tracy, 1955. Modern methods of Plant analysis. Vol. 3-4. Springer verlag.

Prakash, V., Mishra, P., Mishra, M. 2009, Screening of medicinal plant extracts for antioxidant activity, J. Med. Plant Res. 3:8, 608-612.

Purseglove, J. W. Tropical crops: monocotyledons. Longman Group Ltd., London.1974.

Rahaman CH; Choudhury S and Mandal S. 2008.Studies on foliar epidermal micromorphology and stem xylem elements of Dipteracanthus prostratus and Hygrophila schulli- two medicinal plants of Acanthaceae. J. Botan. Soc. Beng. 62 (1), 33-42.

Raja Shanmukha Rao, S. and Ramayya, N. 1987. Trichome types and their taxonomic importance in the Tiliaceae. Indian J. Bot. 10, 65-73.

Rajagopal, T. 1979. Distributional patterns and taxonomic importance of foliar stomata. Indian J. Bot. 2, 63-69.

Saha, S. and Rahaman, C.H. 2013. Pharmacognostic and anatomical studies of Antigonon leptopus Hook. \& Arn. - A promising medicinal climber. Intl. J. Res. Ayurveda \& Pharmacy. 4(2), 186- 191.

Sinha, S., Sharma, A., Reddy, H., Rathi, B., Prasad, N.V.S.R.K., and Vashishtha, A. 2013. Evaluation of phytochemical and pharmacological aspects of 
Holarrhena antidysenterica (Wall.): A comprehensive review; J. Pharmacy Res. 6, 488 - 492.

Trease, G.E. and Evans, W.C.1983. Pharmacognosy, English Language Book Society, Bailliere, Tindall, Germany. Pp 68-73.

trichomes in relation to taxonomy II. Caesalpiniodae. Indian J. For. 6, 4356.
WHO, IUCN, and WWF. 1993. Guideline on the conservation of medicinal plants, (IUCN), Gland Switzerland.

Wilken, R., Veena,

M.S., Wang, M.B., Srivatsan, E.S. 2011.Curcumin: A review of anti-cancer properties and therapeutic activity in head and neck squamous cell carcinoma. Mol. Cancer.10 (12), 23-37.

\section{How to cite this article:}

Jaladhar Pal, Kumaresh Pal and Nirmalya Banerjee. 2020. Pharmacognostic Studies of Selected Species of Curcuma L. a Medicinally Important Member of the Family Zingiberaceae. Int.J.Curr.Microbiol.App.Sci. 9(05): 1831-1845. doi: https://doi.org/10.20546/ijcmas.2020.905.208 